TRANSACTIONS OF THE

AMERICAN MATHEMATICAL SOCIETY

Volume 356, Number 9, Pages 3751-3767

S 0002-9947(04)03439-7

Article electronically published on January 13, 2004

\title{
A CLASS OF PROCESSES ON THE PATH SPACE OVER A COMPACT RIEMANNIAN MANIFOLD WITH UNBOUNDED DIFFUSION
}

\author{
JÖRG-UWE LÖBUS
}

\begin{abstract}
A class of diffusion processes on the path space over a compact Riemannian manifold is constructed. The diffusion of such a process is governed by an unbounded operator. A representation of the associated generator is derived and the existence of a certain local second moment is shown.
\end{abstract}

\section{INTRODUCTION AND BASIC NOTATION}

Infinite dimensional diffusion processes have been studied from several points of view. For example, S. Kusuoka [10, introduced diffusion type Dirichlet forms on Banach spaces. The existence of associated processes is then obtained by using regularity arguments. On the other hand, M. Röckner and T.S. Zhang [16] and A. Eberle [6] used finite dimensional approximation methods to treat infinite dimensional diffusion processes. In these papers, the diffusion is governed by bounded operators.

In contrast, we show the existence of a class of processes with unbounded diffusion operators. For this, we use methods and results of modern Dirichlet form theory (N. Bouleau and F. Hirsch [3], B.K. Driver and M. Röckner [5], M. Fukushima, Y. Oshima, and M. Takeda [8], Z.M. Ma and M. Röckner [13]). The basic structure of a diffusion form we deal with is

$$
\mathcal{E}(F, F):=\int\langle\mathbf{D} F, \mathbf{A D} F\rangle_{\mathbb{H}} d \nu, \quad F \in D(\mathcal{E}),
$$

where $\mathbb{H}$ is the Cameron-Martin space, $\mathbf{D}$ denotes the corresponding gradient operator, and $\nu$ is the Wiener measure on the space $\mathbf{P}_{m_{0}}(M)$ of all Brownian paths $\gamma$ on the compact Riemannian manifold $M$ with $\gamma(0)=m_{0} \in M$. In our setting, the diffusion operator $\mathbf{A}: L^{2}\left(\mathbf{P}_{m_{0}}(M) \rightarrow \mathbb{H}, \nu\right) \supseteq D(\mathbf{A}) \rightarrow L^{2}\left(\mathbf{P}_{m_{0}}(M) \rightarrow \mathbb{H}, \nu\right)$ is unbounded. Let us, however, mention that there are authors speaking in quite different situations of unbounded diffusion coefficients, namely when omitting the operator $\mathbf{A}$ and replacing the measure $d \nu$ with $C d \nu$ where $C$ is a possibly unbounded densitiy function (see, e.g., S. Aida [1]).

Received by the editors October 1, 2002 and, in revised form, June 15, 2003.

2000 Mathematics Subject Classification. Primary 60J60; Secondary 58J65.

Key words and phrases. Path space over a compact Riemannian manifold, diffusion process, unbounded diffusion, generator, local second moment.

This work was carried out while the author was a visitor of the Department of Mathematics at Northwestern University, Evanston, Illinois. 
We verify closability (Section 2$)$ as well as quasi-regularity which implies the existence of an associated right process $\mathbf{M}=\left(\Omega, \mathcal{F},\left(X_{t}\right)_{t \geq 0},\left(P_{\gamma}\right)_{\gamma \in \mathbf{P}_{m_{0}}(M)}\right)$ (Section $3)$. Furthermore, we provide a representation of the associated generator $A$ (Section 4). In particular, the fact that a certain subspace of the space of the cylindrical functions over $\mathbf{P}_{m_{0}}(M)$ is a subset of the domain of $A$ is used to determine the following local second moment,

$$
\begin{gathered}
\lim _{t \rightarrow 0} \frac{1}{t} \int \sum_{v=1}^{N}\left\|x^{v}(\gamma(\cdot))-x^{v}(\tau(\cdot))\right\|_{L^{2}([0,1], d s)}^{2} P_{\tau}\left(X_{t} \in d \gamma\right) \\
=\sum_{v=1}^{N} \int_{s \in[0,1]} \Gamma\left(x^{v}(\tau(s)), x^{v}(\tau(s))\right) d s,
\end{gathered}
$$

weakly in $L^{1}\left(\mathbf{P}_{m_{0}}(M), \nu\right)$ (Section 5$)$. Here, $M$ is considered as isometrically embedded in some $\mathbb{R}^{N}$ and $x^{v}(p), v \in\{1, \ldots, N\}$, denote the standard coordinates of $p \in M$ embedded in $\mathbb{R}^{N}$; finally, $\Gamma$ is the carré du champ operator corresponding to $(\mathcal{E}, D(\mathcal{E}))$.

Let $M$ be a compact connected Riemannian manifold of dimension $d$ without boundary, isometrically embedded in some $\mathbb{R}^{N}$. Let $T_{m}$ denote the tangent space to $M$ at $m(\in M)$ and let $\langle., .\rangle_{T_{m}}$ denote the inner product on $T_{m}$. We fix a covariant derivative $\nabla$ compatible with the underlying Riemannian metric and assume that $\nabla$ is torsion skew symmetric, which means that, if $T$ is the torsion tensor of $\nabla$, then $\langle T\langle\xi, \eta\rangle, \eta\rangle \equiv 0$ for all vector fields $\xi$ and $\eta$ on $M$. This convention guarantees compatibility with the works of B.K. Driver [4], B.K. Driver and M. Röckner [5], and E.P. Hsu [9].

Let $O(M)$ denote the orthonormal frame bundle with respect to $M$. Furthermore, we denote the canonical projection $O(M) \rightarrow M$ by $\pi$ and the canonical horizontal vector fields by $H_{1}, \ldots, H_{d}$. Let $X$ be the space of all Brownian trajectories on $[0,1]$ with values in $\mathbb{R}^{d}$. For fixed $m_{0} \in M$, we introduce the path space $\mathbf{P}_{m_{0}}(M)$ by

$$
\mathbf{P}_{m_{0}}(M):=\left\{\gamma \in C([0,1] \rightarrow M): \gamma(0)=m_{0}\right\}
$$

and equip it with the topology of uniform convergence. Let $\mu$ denote the Wiener measure on $X$ and let $r_{0} \in O(M)$ such that $\pi\left(r_{0}\right)=m_{0}$. According to J. Eells and D. Elworthy [7] and P. Malliavin [12], the solution $r_{x}$ to the Stratonovich SDE

$$
\left\{\begin{aligned}
\partial r_{x}(t) & =\sum_{i=1}^{d} H_{i}\left(r_{x}(t)\right) \partial x_{i}(t), \quad t \in[0,1], \\
r_{x}(0) & =r_{0},
\end{aligned}\right.
$$

$x=\left(x_{1}, \ldots, x_{d}\right) \in X$, defines ( $\mu$-a.e.) a mapping $I: X \rightarrow \mathbf{P}_{m_{0}}(M)$ by $I(x)(t):=$ $\pi\left(r_{x}(t)\right), x \in X, t \in[0,1]$. Considering, simultaneously, $x$ as a $d$-dimensional standard Brownian motion, $I(x)$ becomes a Brownian motion on $M$ whose law on $\mathbf{P}_{m_{0}}(M)$ (the Wiener measure on $\mathbf{P}_{m_{0}}(M)$ ) is denoted by $\nu$.

Finally, as discussed in [9], Section 4, there is an inverse map $L$ of $I$ in the sense that $L \circ I=$ identity $\mu$-a.e. on $X$ and $I \circ L=$ identity $\nu$-a.e. on $\mathbf{P}_{m_{0}}(M)$. Note that, for $x \in X$ and $\gamma \in \mathbf{P}_{m_{0}}(M)$ with $\gamma=I(x)$ and $x=L(\gamma)$, the path $r_{x}$ in $O(M)$ is well defined and that, for $a \in \mathbb{R}^{d}$ and $0 \leq s, t \leq 1$,

$$
\left\langle r_{x}(s) a, r_{x}(s) a\right\rangle_{T_{\gamma(s)}}=\left\langle r_{x}(t) a, r_{x}(t) a\right\rangle_{T_{\gamma(t)}}=|a|_{\mathbb{R}^{d}}^{2} .
$$


The parallel transport from $T_{\gamma(s)}$ to $T_{\gamma(t)}$ along $\gamma \in \mathbf{P}_{m_{0}}(M)$ is $\nu$-a.e. defined as follows. For $x \in X$ and $\gamma \in \mathbf{P}_{m_{0}}(M)$ with $\gamma=I(x)$ and $x=L(\gamma)$, set

$$
\mathcal{T}_{t \leftarrow s}^{\gamma}:=r_{x}(t) r_{x}^{-1}(s), \quad 0 \leq s, t \leq 1 .
$$

Introduce the abbreviation $L^{p}(\nu)$ for $L^{p}\left(\mathbf{P}_{m_{0}}(M), \nu\right), 1 \leq p \leq \infty$, and define the set of all cylindrical functions on $\mathbf{P}_{m_{0}}(M)$,

$$
\begin{aligned}
& Z:=\left\{F(\gamma)=f\left(\gamma\left(s_{1}\right) ; \ldots ; \gamma\left(s_{k}\right)\right), \gamma \in \mathbf{P}_{m_{0}}(M):\right. \\
& \left.\quad 0<s_{1}<\ldots<s_{k}=1, f \in C^{\infty}\left(M^{k}\right), k \in \mathbb{N}\right\} .
\end{aligned}
$$

Set

$$
\begin{aligned}
Y:=\left\{F(\gamma)=f\left(\gamma\left(s_{1}\right) ; \ldots ; \gamma\left(s_{k}\right)\right), \gamma \in \mathbf{P}_{m_{0}}(M):\right. & \\
& \left.F \in Z, s_{1}, \ldots, s_{k} \in\left\{\frac{l}{2^{n}}: l \in\left\{1, \ldots, 2^{n}\right\}, n \in \mathbb{N}\right\}\right\} .
\end{aligned}
$$

As $Z$ is dense in $L^{2}(\nu)$ (see [5]), $Y$ is also dense in $L^{2}(\nu)$. Let $\left(e_{j}\right)_{j=1, \ldots, d}$ be a standard basis in $\mathbb{R}^{d}$ and let

$$
\begin{aligned}
H_{1}(t) & =1, \quad t \in[0,1], \\
H_{2^{m}+k}(t) & =\left\{\begin{aligned}
2^{\frac{m}{2}} & \text { if } t \in\left[\frac{k-1}{2^{m}}, \frac{2 k-1}{2^{m+1}}\right), \\
-2^{\frac{m}{2}} & \text { if } t \in\left[\frac{2 k-1}{2^{m+1}}, \frac{k}{2^{m}}\right), \\
0 & \text { otherwise, }
\end{aligned}\right.
\end{aligned}
$$

denote the system of the Haar functions on $[0,1]$. Furthermore, define

$$
g_{d(r-1)+j}:=H_{r} \cdot e_{j}, \quad r \in \mathbb{N}, j \in\{1, \ldots, d\} .
$$

As the system of the Haar functions $\left(H_{n}\right)_{n \in \mathbb{N}}$ is complete in $L^{2}([0,1] \rightarrow \mathbb{R}, d s)$, the system $\left(g_{n}\right)_{n \in \mathbb{N}}$ is complete in $L^{2}\left([0,1] \rightarrow \mathbb{R}^{d}, d s\right)$. Therefore, $\left(S_{n}\right)_{n \in \mathbb{N}}$, defined by

$$
S_{n}(s):=\int_{0}^{s} g_{n}(u) d u, \quad s \in[0,1], n \in \mathbb{N},
$$

is complete in the Cameron-Martin space $\mathbb{H}$, the space of all $\mathbb{R}^{d}$-valued absolutely continuous functions $h$ on $[0,1]$ with $h(0)=0$ endowed with the norm

$$
|h|_{\mathbb{H}}:=\left(\int_{0}^{1}\left|h^{\prime}(s)\right|_{\mathbb{R}^{d}}^{2} d s\right)^{\frac{1}{2}} .
$$

\section{Definition of the Form AND Closability}

For $F \in Y$ and $\nu$-a.e. $\gamma \in \mathbf{P}_{m_{0}}(M)$, define

$$
D_{s} F(\gamma):=\sum_{i=1}^{k} \chi_{\left[0, s_{i}\right]}(s)\left\{\mathcal{T}_{s \leftarrow s_{i}}^{\gamma}\left(\nabla_{s_{i}} f\right)(\underline{\gamma})\right\}, \quad s \in[0,1],
$$

where $\left(\nabla_{s_{i}} f\right)(\underline{\gamma}) \equiv\left(\nabla_{s_{i}} f\right)\left(\gamma\left(s_{1}\right) ; \ldots ; \gamma\left(s_{k}\right)\right) \in T_{\gamma\left(s_{i}\right)}$ denotes the gradient of the function $f$ relative to the $i$-th variable while holding the remaining variables fixed. 
Here, $f$ and the $s_{1}, \ldots, s_{k}$ are as in the definition of $Z$. Furthermore, for $F \in Z$ and

$$
\begin{aligned}
\mathbf{D}_{s} F(\gamma) & :=\int_{0}^{s} r_{L(\gamma)}^{-1}\left(s^{\prime}\right) D_{s^{\prime}} F(\gamma) d s^{\prime} \\
& =\sum_{i=1}^{k} s \wedge s_{i} \cdot r_{L(\gamma)}^{-1}\left(s_{i}\right)\left(\nabla_{s_{i}} f\right)(\underline{\gamma}), \quad s \in[0,1], \gamma \in \mathbf{P}_{m_{0}}(M),
\end{aligned}
$$

we have $\mathbf{D} F \in \mathbb{H} \nu$-a.e. See also (2.1).

Any $F \in Y$ has the representation $F(\gamma)=f\left(\gamma\left(s_{1}\right) ; \ldots ; \gamma\left(s_{k}\right)\right)$ where $s_{1}=$ $\frac{l_{1}}{2^{n^{\prime}}}, \ldots, s_{k}=\frac{l_{k}}{2^{n^{\prime}}}$, for some $k \in \mathbb{N}, n^{\prime} \in \mathbb{N}, l_{1}, \ldots, l_{k} \in\left\{1, \ldots, 2^{n^{\prime}}\right\}$, and $f \in$ $C^{\infty}\left(M^{k}\right)$. As $H_{2^{m}+l}(t)=0$ on $[0,1] \backslash\left[\frac{l-1}{2^{m}}, \frac{l}{2^{m}}\right], \int_{(l-1) / 2^{m}}^{l / 2^{m}} H_{2^{m}+l}(t) d t=0$, and either $\left(\frac{l-1}{2^{m}}, \frac{l}{2^{m}}\right) \subseteq\left[0, s_{i}\right]$ or $\left(\frac{l-1}{2^{m}}, \frac{l}{2^{m}}\right) \subseteq\left(s_{i}, 1\right]$ if $m \geq n^{\prime}, l \in\left\{1, \ldots, 2^{m}\right\}$, and $i \in\{1, \ldots, k\}$, from (2.2), we obtain the following lemma which is crucial for the technical procedure.

Lemma 2.1. Let $F \in Y$. There exists $n_{0} \in \mathbb{N}$ such that, for $\nu$-a.e. $\gamma \in \mathbf{P}_{m_{0}}(M)$,

$$
\left\langle S_{n}, \mathbf{D} F(\gamma)\right\rangle_{\mathbb{H}}=0, \quad n>n_{0} .
$$

Let us define the diffusion operator we are dealing with in this paper. Choose an increasing sequence $\left(\lambda_{n}\right)_{n \in \mathbb{N}}$ of positive real numbers and define the operator

$$
\begin{aligned}
D(\mathbf{A}) & :=\left\{\Phi \in L^{2}\left(\mathbf{P}_{m_{0}}(M) \rightarrow \mathbb{H}, \nu\right): \int \sum_{i=1}^{\infty} \lambda_{i}^{2}\left\langle S_{i}, \Phi\right\rangle_{\mathbb{H}}^{2} d \nu<\infty\right\}, \\
\mathbf{A} \Phi(\gamma) & :=\sum_{i=1}^{\infty} \lambda_{i}\left\langle S_{i}, \Phi(\gamma)\right\rangle_{\mathbb{H}} S_{i}, \gamma \in \mathbf{P}_{m_{0}}(M), \quad \Phi \in D(\mathbf{A}),
\end{aligned}
$$

mapping $L^{2}\left(\mathbf{P}_{m_{0}}(M) \rightarrow \mathbb{H}, \nu\right) \supseteq D(\mathbf{A}) \rightarrow L^{2}\left(\mathbf{P}_{m_{0}}(M) \rightarrow \mathbb{H}, \nu\right)$. For $F \in Y$, we have $\int\left\langle S_{i}, \mathbf{D} F\right\rangle_{\mathbb{H}}^{2} d \nu\left\langle\infty, i \in \mathbb{N}\right.$. By Lemma 2.1, for $F \in Y$, there is $n_{0} \in \mathbb{N}$ such that, for all $i>n_{0}$, it holds that $\int\left\langle S_{i}, \mathbf{D} F\right\rangle_{\mathbb{H}}^{2} d \nu=0$. Therefore, we obtain $\{\mathbf{D} F: F \in Y\} \subseteq D(\mathbf{A})$. Furthermore, for all $F \in Y$, we get

$$
\begin{aligned}
\int\langle\mathbf{D} F, \mathbf{A D} F\rangle_{\mathbb{H}} d \nu & =\int\left\langle\mathbf{D} F, \sum_{i=1}^{\infty} \lambda_{i}\left\langle S_{i}, \mathbf{D} F\right\rangle_{\mathbb{H}} S_{i}\right\rangle_{\mathbb{H}} d \nu \\
& =\sum_{i=1}^{\infty} \lambda_{i} \int\left\langle S_{i}, \mathbf{D} F\right\rangle_{\mathbb{H}}^{2} d \nu \\
& <\infty .
\end{aligned}
$$

Consequently, the nonnegative symmetric bilinear form

$$
\begin{aligned}
\mathcal{E}(F, F) & :=\int\langle\mathbf{D} F, \mathbf{A} \mathbf{D} F\rangle_{\mathbb{H}} d \nu \\
& =\int\left|\mathbf{A}^{1 / 2} \mathbf{D} F\right|_{\mathbb{H}}^{2} d \nu, \quad F \in Y,
\end{aligned}
$$

is well defined.

Remarks. (1) It is known from [5], Lemma 3, or [9], Proposition 5.3, that the operator $\mathbf{D}: Z \rightarrow L^{2}\left(\mathbf{P}_{m_{0}}(M) \rightarrow \mathbb{H}, \nu\right)$ is closable on $L^{2}(\nu)$. Let $(\mathbf{D}, D(\mathbf{D}))$ denote 
this closure. Equivalently, the Ornstein-Uhlenbeck form

$$
\mathcal{E}^{O U}(F, F):=\int|\mathbf{D} F|_{\mathbb{H}}^{2} d \nu, \quad F \in Z
$$

is closable on $L^{2}(\nu)$. For its closure $\left(\mathcal{E}^{O U}, D\left(\mathcal{E}^{O U}\right)\right)$, we have $D(\mathbf{D})=D\left(\mathcal{E}^{O U}\right)$.

(2) Fix $\gamma \in \mathbf{P}_{m_{0}}(M)$ and consider, for this remark, $\mathbf{A}$ as an operator mapping $D(\mathbf{A}) \supseteq \mathbb{H} \rightarrow \mathbb{H}$. S. Albeverio and M. Röckner 2, for example, suggest in a similar situation to reduce a form of type (2.4) to a more simple one by choosing another Hilbert space $\left(H,\langle\cdot, \cdot\rangle_{H}\right):=\left(D\left(\mathbf{A}^{1 / 2}\right),\left\langle\mathbf{A}^{1 / 2} \cdot \mathbf{A}^{1 / 2} \cdot\right\rangle_{\mathbb{H}}\right)$. The price one has to pay is that the classical relation between directional derivative $\partial_{h}$ and gradient $\mathbf{D}$, namely $\partial_{h} F=\langle\mathbf{D} F, h\rangle_{H}$, is in general not satisfied anymore if $\partial_{h} F=\langle\mathbf{D} F, h\rangle_{\mathbb{H}}$.

Theorem 2.2. The bilinear form $(\mathcal{E}, Y)$ is closable on $L^{2}(\nu)$.

Proof. Suppose $F_{n} \in Y, n \in \mathbb{N}$, with $F_{n} \underset{n \rightarrow \infty}{\longrightarrow} 0$ in $L^{2}(\nu)$ and $\mathcal{E}\left(F_{n}-F_{m}, F_{n}-F_{m}\right)$ $\underset{m, n \rightarrow \infty}{\longrightarrow}$ 0. In particular, (2.4) implies

$$
\mathbf{A}^{1 / 2} \mathbf{D} F_{n} \underset{n \rightarrow \infty}{\longrightarrow} \Psi \quad \text { in } \quad L^{2}\left(\mathbf{P}_{m_{0}}(M) \rightarrow \mathbb{H}, \nu\right)
$$

for some $\Psi \in L^{2}\left(\mathbf{P}_{m_{0}}(M) \rightarrow \mathbb{H}, \nu\right)$. Define

$$
\mathbf{J} F:=\sum_{i=1}^{\infty} \lambda_{i}^{-1 / 2}\left\langle S_{i}, F\right\rangle_{\mathbb{H}} S_{i}, \quad F \in L^{2}\left(\mathbf{P}_{m_{0}}(M) \rightarrow \mathbb{H}, \nu\right)
$$

Since $\mathbf{J}$ is a bounded operator on $L^{2}\left(\mathbf{P}_{m_{0}}(M) \rightarrow \mathbb{H}, \nu\right)$, we verify

$$
\mathbf{D} F_{n}=\mathbf{J A}^{1 / 2} \mathbf{D} F_{n} \underset{n \rightarrow \infty}{\longrightarrow} \mathbf{J} \Psi \quad \text { in } \quad L^{2}\left(\mathbf{P}_{m_{0}}(M) \rightarrow \mathbb{H}, \nu\right)
$$

from (2.5). As $(\mathbf{D}, Z)$ is closable on $L^{2}(\nu)$, we obtain $\mathbf{J} \Psi=0$. It follows from (2.6) and $\lambda_{i}>0, i \in \mathbb{N}$, that $\Psi=0$. Thus, relation (2.5) leads to $\mathbf{A}^{1 / 2} \mathbf{D} F_{n} \underset{n \rightarrow \infty}{\longrightarrow} 0$ in $L^{2}\left(\mathbf{P}_{m_{0}}(M) \rightarrow \mathbb{H}, \nu\right)$ which implies $\mathcal{E}\left(F_{n}, F_{n}\right)=\int\left|\mathbf{A}^{1 / 2} \mathbf{D} F_{n}\right|_{\mathbb{H}}^{2} d \nu \underset{n \rightarrow \infty}{\longrightarrow} 0$.

Let $(\mathcal{E}, D(\mathcal{E}))$ denote the closure of $(\mathcal{E}, Y)$ on $L^{2}(\nu)$.

Remark. (3) Let $F \in D(\mathcal{E})$ and let $F_{n} \in Y, n \in \mathbb{N}$, be a sequence converging to $F$ in $\mathcal{E}_{1}^{1 / 2}=\left(\|\cdot\|_{L^{2}(\nu)}^{2}+\mathcal{E}(\cdot, \cdot)\right)^{1 / 2}$-norm. Since $\lambda_{i}>0, i \in \mathbb{N}$, is an increasing sequence of positive real numbers and $(\mathcal{E}, Y)=\left(\mathcal{E}^{O U}, Y\right)$ if $\lambda_{i}=1, i \in \mathbb{N}$, from (2.3) it follows that $F_{n}, n \in \mathbb{N}$, is a Cauchy sequence in $\left(\mathcal{E}^{O U}\right)_{1}^{1 / 2}=\left(\|\cdot\|_{L^{2}(\nu)}^{2}+\mathcal{E}^{O U}(\cdot, \cdot)\right)^{1 / 2}$-norm. Therefore, $F_{n} \underset{n \rightarrow \infty}{\longrightarrow} F$ in $\left(\mathcal{E}^{O U}\right)_{1}^{1 / 2}$-norm. Thus, we have $D(\mathcal{E}) \subseteq D\left(\mathcal{E}^{O U}\right)=D(\mathbf{D})$. Since, by self-adjointness, $\mathbf{A}^{1 / 2}$ is a closed operator, it holds that $\{\mathbf{D} F: F \in$ $D(\mathcal{E})\} \subseteq D\left(\mathbf{A}^{1 / 2}\right)$ and relations 2.3 and 2.4 yield

$$
\begin{aligned}
\mathcal{E}(F, F) & =\sum_{i=1}^{\infty} \lambda_{i} \int\left\langle S_{i}, \mathbf{D} F\right\rangle_{\mathbb{H}}^{2} d \nu \\
& =\int\left|\mathbf{A}^{1 / 2} \mathbf{D} F\right|_{\mathbb{H}}^{2} d \nu, \quad F \in D(\mathcal{E}) .
\end{aligned}
$$




\section{QUASI-REgUlarity AND ASSOCIATED PROCESS}

Let $h \in \mathbb{H}, t \in \mathbb{R}, s \in[0,1]$, and $\gamma \in \mathbf{P}_{m_{0}}(M)$ and let $\sigma$ denote the solution to the geometric flow equation

$$
\left\{\begin{aligned}
\dot{\sigma}^{h}(t, s)(\gamma) & =\mathcal{T}_{s \leftarrow 0}^{\sigma^{h}(t, \cdot)(\gamma)} r_{0} h(s), \\
\sigma^{h}(0, s)(\gamma) & =\gamma(s)
\end{aligned}\right.
$$

Note that " " stands for differentiation with respect to $t$. In particular, we have $\sigma^{h}(\cdot, s)(\gamma) \in C^{1}(\mathbb{R} \rightarrow M), \sigma^{h}(t)(\gamma) \equiv \sigma^{h}(t, \cdot)(\gamma) \in \mathbf{P}_{m_{0}}(M)$. For $h \in \mathbb{H}$ and $\nu$-a.e. $\gamma \in \mathbf{P}_{m_{0}}(M)$, there exists a unique solution (see 4 ] and 9$]$ ). For $F \in Y$ given as in (1.1), the directional derivative along the direction $h \in \mathbb{H}$ satisfies

$$
\begin{aligned}
\partial_{h} F & :=\lim _{t \rightarrow 0} \frac{F\left(\sigma^{h}(t)\right)-F}{t} \\
& =\sum_{i=1}^{k}\left\langle\nabla_{s_{i}} f, \dot{\sigma}^{h}\left(0, s_{i}\right)\right\rangle_{T_{\cdot\left(s_{i}\right)}} \\
& =\sum_{i=1}^{k}\left\langle\nabla_{s_{i}} f, \mathcal{T}_{s_{i} \leftarrow 0} r_{0} h\left(s_{i}\right)\right\rangle_{T_{\cdot\left(s_{i}\right)}} \\
& =\sum_{i=1}^{k}\left\langle\mathcal{T}_{0 \leftarrow s_{i}}\left(\nabla_{s_{i}} f\right), r_{0} h\left(s_{i}\right)\right\rangle_{T_{m_{0}}} \\
& =\langle\mathbf{D} F, h\rangle_{\mathbb{H}} \quad \nu \text {-a.e. }
\end{aligned}
$$

See also (2.2).

Remark. (4) For every $h \in \mathbb{H}$, the operator $\partial_{h}: Z \rightarrow L^{2}(\nu)$ is closable on $L^{2}(\nu)$. Let $\left(\partial_{h}, D\left(\partial_{h}\right)\right)$ denote the corresponding closure. It holds that $D(\mathcal{E}) \subseteq D(\mathbf{D}) \subseteq D\left(\partial_{h}\right)$, $h \in \mathbb{H}$, and $\partial_{h} F=\langle\mathbf{D} F, h\rangle_{\mathbb{H}}, F \in D(\mathbf{D})$; cf. 9, Theorem 5.2 and Proposition 5.3. Therefore,

$$
\mathcal{E}(F, F)=\sum_{i=1}^{\infty} \lambda_{i} \int\left(\partial_{S_{i}} F\right)^{2} d \nu, \quad F \in D(\mathcal{E}) .
$$

Proposition 3.1. The form $(\mathcal{E}, D(\mathcal{E}))$ is a Dirichlet form on $L^{2}(\nu)$.

Proof. We have

$$
\begin{aligned}
\mathcal{E}(F, F) & =\sum_{i=1}^{\infty} \lambda_{i} \int\left(\partial_{S_{i}} F\right)^{2} d \nu \\
& =\sum_{i=1}^{\infty} \lambda_{i} \int\left(\left.\frac{d}{d t}\right|_{0} F\left(\sigma^{S_{i}}(t)\right)\right)^{2} d \nu, \quad F \in Y .
\end{aligned}
$$

It follows directly from [13], Proposition I, 4.10, and the chain rule that $(\mathcal{E}, D(\mathcal{E}))$ is a Dirichlet form.

An important tool for the subsequent technical procedure will be the following assertion; cf. [13], Chapter IV, Lemma 4.1. Note that, for $u, v \in D(\mathcal{E})$, we have $u \vee v \in D(\mathcal{E}) \subseteq D(\mathbf{D}) \subseteq D\left(\partial_{S_{i}}\right), i \in \mathbb{N}$ (see Remarks $(3)$ and $\left.(4)\right)$. 
Lemma 3.2. Let $u, v \in D(\mathcal{E})$. For all $i \in \mathbb{N}$, we have

$$
\partial_{S_{i}}(u \vee v)=\chi_{\{u>v\}} \partial_{S_{i}} u+\chi_{\{u<v\}} \partial_{S_{i}} v+\frac{1}{2} \chi_{\{u=v\}}\left(\partial_{S_{i}} u+\partial_{S_{i}} v\right) \quad \nu \text {-a.e. }
$$

Proof. Having representation (3.2) of $(\mathcal{E}, Y)$ in mind, the proof can be obtained from that of [13], Chapter IV, Lemma 4.1 by replacing therein $\frac{\partial}{\partial k}$ with $\partial_{S_{i}}$ and $\mathcal{F} C_{b}^{\infty}$ with $Y$.

\section{Proposition 3.3. Suppose}

$$
\lambda_{n} \leq c n^{1-\varepsilon}, \quad n \in \mathbb{N}, \quad \text { for some } c>0 \text { and } \varepsilon \in(0,1) .
$$

Then the Dirichlet form $(\mathcal{E}, D(\mathcal{E}))$ is quasi-regular.

Proof. In steps 1-3 below, we show that there is an $\mathcal{E}$-nest consisting of compact sets.

Step 1. For $r \in \mathbb{N}, l \in\left\{0, \ldots, 2^{r-1}-1\right\}$, and $k=2^{r-1}+l$, set $s_{k}:=(2 l+1) 2^{-r}$. Let $x^{v}(p)$ denote the standard coordinates of $p \in M$ embedded in $\mathbb{R}^{N}, v \in$ $\{1, \ldots, N\}$. Fix $\tau \in \mathbf{P}_{m_{0}}(M), k=2^{r-1}+l$, and $v \in\{1, \ldots, N\}$. Consider the functions $f_{v, k, \tau}(p):=x^{v}(p)-x^{v}\left(\tau\left(s_{k}\right)\right), p \in M$, and

$$
F_{v, k, \tau}(\gamma):=f_{v, k, \tau}\left(\gamma\left(s_{k}\right)\right)=x^{v}\left(\gamma\left(s_{k}\right)\right)-x^{v}\left(\tau\left(s_{k}\right)\right), \quad \gamma \in \mathbf{P}_{m_{0}}(M) ;
$$

obviously, $F_{v, k, \tau}$ belongs to $Y$. Furthermore, let either $i=j$ or $i=d\left(2^{m}+u-1\right)+j$ for some $m \in\{0,1, \ldots\}, u \in\left\{1, \ldots, 2^{m}\right\}$ and $j \in\{1, \ldots, d\}$. We have

$$
\begin{aligned}
\left|\left\langle S_{i}, \mathbf{D} F_{v, k, \tau}(\gamma)\right\rangle_{\mathbb{H}}\right| & =\left|\left\langle\mathcal{T}_{0 \leftarrow s_{k}}^{\gamma} \nabla_{s_{k}} x^{v}\left(\gamma\left(s_{k}\right)\right), r_{0} S_{i}\left(s_{k}\right)\right\rangle_{T_{m_{0}}}\right| \\
& \leq\left\|\mathcal{T}_{0 \leftarrow s_{k}}^{\gamma} \nabla_{s_{k}} x^{v}\left(\gamma\left(s_{k}\right)\right)\right\|_{T_{m_{0}}}\left\|r_{0} S_{i}\left(s_{k}\right)\right\|_{T_{m_{0}}} \\
& =\left\|\nabla_{s_{k}} x^{v}\left(\gamma\left(s_{k}\right)\right)\right\|_{T_{\gamma\left(s_{k}\right)}}\left|S_{i}\left(s_{k}\right)\right|_{\mathbb{R}^{d}} \\
& =\left\|\nabla_{s_{k}} x^{v}\left(\gamma\left(s_{k}\right)\right)\right\|_{T_{\gamma\left(s_{k}\right)}}\left|\left(S_{i}\left(s_{k}\right)\right)^{j}\right|
\end{aligned}
$$

for $\nu$-a.e. $\gamma \in \mathbf{P}_{m_{0}}(M)$. As mentioned in [5], proof of Proposition 5, $\left\|\nabla x^{v}(p)\right\|_{T_{p}}$, $p \in M$, is bounded by some constant $K$ since $M$ is compact. Furthermore, the definitions of $s_{k}$ and $S_{i}$ yield $\left|\left(S_{i}\left(s_{k}\right)\right)^{j}\right| \leq 1$ if $i=j$ for some $j \in\{1, \ldots, d\}$. Moreover, $\left|\left(S_{i}\left(s_{k}\right)\right)^{j}\right| \leq 2^{-(m / 2+1)}$ if $i=d\left(2^{m}+u-1\right)+j$ for some $m \in\{0,1, \ldots\}$, $u \in\left\{1, \ldots, 2^{m}\right\}, j \in\{1, \ldots, d\}$, and $m<r$ as well as $s_{k}=(2 l+1) 2^{-r} \in\left(\frac{u-1}{2^{m}}, \frac{u}{2^{m}}\right)$. Otherwise, we have $S_{i}\left(s_{k}\right)=0$. Therefore, 3.5) implies

$$
\begin{aligned}
\sum_{i=1}^{\infty} \lambda_{i} & \left(\partial_{S_{i}} F_{v, k, \tau}(\gamma)\right)^{2}=\sum_{i=1}^{\infty} \lambda_{i}\left\langle S_{i}, \mathbf{D} F_{v, k, \tau}(\gamma)\right\rangle_{\mathbb{H}}^{2} \\
= & \sum_{j=1}^{d} \lambda_{j}\left\langle S_{j}, \mathbf{D} F_{v, k, \tau}(\gamma)\right\rangle_{\mathbb{H}}^{2} \\
& \quad+\sum_{m=0}^{\infty} \sum_{u=1}^{2^{m}} \sum_{j=1}^{d} \lambda_{d\left(2^{m}+u-1\right)+j}\left\langle S_{d\left(2^{m}+u-1\right)+j}, \mathbf{D} F_{v, k, \tau}(\gamma)\right\rangle_{\mathbb{H}}^{2} \\
\leq & K^{2} d \lambda_{d}+K^{2} d \sum_{m=0}^{\infty} \lambda_{d 2^{m+1}} 2^{-(m+2)}
\end{aligned}
$$


for $\nu$-a.e. $\gamma \in \mathbf{P}_{m_{0}}(M)$. Finally, from (3.3), we obtain

$$
\sum_{i=1}^{\infty} \lambda_{i}\left(\partial_{S_{i}} F_{v, k, \tau}(\gamma)\right)^{2} \leq K^{2} c d^{2-\varepsilon} \frac{2^{1+\varepsilon}-1}{2^{1+\varepsilon}-2}=: C_{1}
$$

for $\nu$-a.e. $\gamma \in \mathbf{P}_{m_{0}}(M)$ and

$$
\mathcal{E}\left(F_{v, k, \tau}, F_{v, k, \tau}\right) \leq C_{1},
$$

where the right-hand side is independent of $k$ (resp. $s_{k}$ ), $v \in\{1, \ldots, N\}$, and $\tau$.

Step 2. We apply a method introduced in [5] and [15]. Set

$$
G_{n, \tau}:=\sup _{\substack{k \in\{1, \ldots, n\} \\ v \in\{1, \ldots, N\}}}\left|F_{v, k, \tau}\right|, \quad n \in \mathbb{N} .
$$

It follows now from (3.7), relation

$$
\mathcal{E}\left(G_{n, \tau}, G_{n, \tau}\right)=\int \sum_{i=1}^{\infty} \lambda_{i}\left(\partial_{S_{i}} G_{n, \tau}(\gamma)\right)^{2} d \nu
$$

and Lemma 3.2 that

$$
\mathcal{E}\left(G_{n, \tau}, G_{n, \tau}\right) \leq C_{1}, \quad n \in \mathbb{N}
$$

Since $M$ is compact, there exists $C_{2}>0$, such that

$$
\left|x^{v}(p)\right| \leq \frac{1}{2} \sqrt{C_{2}}, \quad p \in M, v \in\{1, \ldots, N\} .
$$

From (3.4) and the definition of $G_{n, \tau}$ it follows that $\left\|G_{n, \tau}\right\|_{L^{2}(\nu)}^{2} \leq C_{2}$ and, thus,

$$
\mathcal{E}_{1}\left(G_{n, \tau}, G_{n, \tau}\right) \leq C_{1}+C_{2}=: C_{3}, \quad n \in \mathbb{N} .
$$

Step 3. In this step, we proceed as in [5] and [15]. In particular, we apply the Banach-Saks property of the Hilbert space $\left(D(\mathcal{E}), \mathcal{E}_{1}^{1 / 2}\right)$, which states that every bounded sequence in $\left(D(\mathcal{E}), \mathcal{E}_{1}^{1 / 2}\right)$ has a subsequence whose Cesaro means converge strongly (see, for example, [14). Accordingly, relation (3.9) and the fact that the sequence $\left(G_{n, \tau}\right)_{n \in \mathbb{N}}$ satisfies $G_{n, \tau} \leq G_{n+1, \tau}, n \in \mathbb{N}$, imply that the function

$$
H_{\tau}(\gamma):=\sup _{\substack{s \in[0,1] \\ v \in\{1, \ldots, N\}}}\left|x^{v}(\gamma(s))-x^{v}(\tau(s))\right|, \quad \gamma \in \mathbf{P}_{m_{0}}(M),
$$

belongs to $D(\mathcal{E})$ and that

$$
\mathcal{E}_{1}\left(H_{\tau}, H_{\tau}\right) \leq C_{3} .
$$

Let $\left\{\tau_{k}: k \in \mathbb{N}\right\}$ be a dense set in $\mathbf{P}_{m_{0}}(M)$. Set

$$
K_{n}:=\inf _{1 \leq k \leq n} H_{\tau_{k}}, \quad n \in \mathbb{N} .
$$

We have $K_{n} \in D(\mathcal{E})$. Again, recalling the Banach-Saks property of $\left(D(\mathcal{E}), \mathcal{E}_{1}^{1 / 2}\right)$, the last relation implies that $\mathcal{E}_{1}\left(K_{n}, K_{n}\right) \underset{n \rightarrow \infty}{\longrightarrow} 0$. According to [13], Chapter III, Proposition 3.5, there exists a subsequence $K_{n_{k}}, k \in \mathbb{N}$, and an $\mathcal{E}$-nest $F_{m}, m \in \mathbb{N}$, such that $K_{n_{k}}$ converges uniformly to zero (as $k \rightarrow \infty$ ) on each $F_{m}$. Consult also 3], Section I.8. As in [5], proof of Proposition 5, it follows now from the definition of $K_{n}, n \in \mathbb{N}$, that each $F_{m}$ is totally bounded. Thus, $F_{m}, m \in \mathbb{N}$, form an $\mathcal{E}$-nest consisting of compact sets.

Step 4. For fixed $\tau \in \mathbf{P}_{m_{0}}(M)$, the system of functions $F_{v, k, \tau}, v \in\{1, \ldots, N\}$, $k \in \mathbb{N}$, introduced in (3.4) separates the points in $\mathbf{P}_{m_{0}}(M)$. 
Together with Theorem 2.2 and the result of Step 3, quasi-regularity follows now from its definition (see [13], Chapter IV, Definition 3.1).

Proposition 3.4. The form $(\mathcal{E}, D(\mathcal{E}))$ is local.

Proof. We follow [5], proof of Proposition 5 (ii), and [13], Example V.1.12. Let $F, G \in D(\mathcal{E}) \cap L^{\infty}(\nu)$ with $\operatorname{supp}[F] \cap \operatorname{supp}[G]=\emptyset$. According to [13], Propositions I.4.17 (i) and V.1.2 (ii), we have to verify $\mathcal{E}(F, G)=0$. Since $\mathcal{E}(F, G)=$ $\int\left\langle\mathbf{A}^{1 / 2} \mathbf{D} F, \mathbf{A}^{1 / 2} \mathbf{D} G\right\rangle_{\mathbb{H}} d \nu$ (cf. (2.7)), it is sufficient to show that

$$
\mathbf{D} F=0 \quad \nu \text {-a.e. on } \mathbf{P}_{m_{0}}(M) \backslash \operatorname{supp}[F] \text {. }
$$

From $D(\mathcal{E}) \subseteq D\left(\mathcal{E}^{O U}\right)$ and [5], equation (11), we obtain

$$
\mathbf{D}(U \cdot V)=U \cdot \mathbf{D}(V)+V \cdot \mathbf{D}(U), \quad U, V \in D(\mathcal{E}) \cap L^{\infty}(\nu) .
$$

See also [13], Example V.1.12. Furthermore, from [13], Proposition V.1.7, we get the existence of $V \in D(\mathcal{E}) \cap L^{\infty}(\nu)$ with $0 \leq V \leq \chi_{\mathbf{P}_{m_{0}}(M) \backslash \operatorname{supp}[F]}$ and $V>0 \nu$-a.e. on $\mathbf{P}_{m_{0}}(M) \backslash \operatorname{supp}[F]$; here $\chi$ denotes the indicator function. Now, relation (3.11) implies

$$
0=F \cdot \mathbf{D}(V)+V \cdot \mathbf{D}(F) \quad \nu \text {-a.e. }
$$

This yields 3.10.

As a consequence of Propositions 3.3 and 3.4, we get with 13, Theorems IV.3.5 and V.1.11:

Theorem 3.5. There exists a diffusion process $\mathbf{M}$ associated with $(\mathcal{E}, D(\mathcal{E}))$.

\section{Generator}

We start with a technical lemma.

Lemma 4.1. Let $F \in Y$ and $n \in \mathbb{N}$. Then the derivatives $\left.\frac{d}{d t}\right|_{0} \partial_{S_{n}} F\left(\sigma^{S_{n}}(-t)\right)$, $\left.\frac{d}{d t}\right|_{0} \frac{d \nu \circ \sigma^{S_{n}}(-t)}{d \nu}$, and $\left.\frac{d}{d t}\right|_{0}\left\{\partial_{S_{n}} F\left(\sigma^{S_{n}}(-t)\right) \frac{d \nu \circ \sigma^{S_{n}}(-t)}{d \nu}\right\}$ exist in $L^{2}(\nu)$ and we have

$$
\begin{aligned}
\left.\frac{d}{d t}\right|_{0} & \left\{\partial_{S_{n}} F\left(\sigma^{S_{n}}(-t)\right) \frac{d \nu \circ \sigma^{S_{n}}(-t)}{d \nu}\right\} \\
& =-\partial_{S_{n}} \partial_{S_{n}} F+\left.\frac{d}{d t}\right|_{0} \frac{d \nu \circ \sigma^{S_{n}}(-t)}{d \nu} \cdot \partial_{S_{n}} F \quad \nu \text {-a.e. }
\end{aligned}
$$

Proof. Step 1. Introduce

$$
\varphi_{t}(\gamma):=\frac{d \nu \circ \sigma^{S_{n}}(-t)(\gamma)}{d \nu}(\gamma), \quad \gamma \in \mathbf{P}_{m_{0}}(M), t \in \mathbb{R}
$$

The existence of $\left.\frac{d}{d t}\right|_{0} \varphi_{t}$ in $L^{2}(\nu)$ is shown in [4], Theorem 8.5 and in the proof of Theorem 9.1. Note that the result for $h \in C^{1}\left([0,1] \rightarrow \mathbb{R}^{d}\right)$ presented in [4] can be extended to general $h \in \mathbb{H}$ by [9], Theorems 3.5 and 4.1 and the proof of Theorem 5.1 .

Step 2. Let $n \in \mathbb{N}$ and let $F \in Y$ be given as in (1.1). According to (3.1), we have, for $\nu$-a.e. $\gamma \in \mathbf{P}_{m_{0}}(M)$,

$$
\begin{aligned}
\psi_{t}(\gamma) & :=\partial_{S_{n}} F\left(\sigma^{S_{n}}(-t)(\gamma)\right) \\
& \left.=\sum_{i=1}^{k}\left\langle\mathcal{T}_{0 \leftarrow s_{i}}^{\sigma^{S_{n}}(-t)(\gamma)}\left(\nabla_{s_{i}} f\right) \underline{\left(\sigma^{S_{n}}(-t)(\gamma)\right.}\right), r_{0} S_{n}\left(s_{i}\right)\right\rangle_{T_{m_{0}}}, \quad t \in \mathbb{R}
\end{aligned}
$$


From $\left|S_{n}(s)\right|_{\mathbb{R}^{d}} \leq 1, s \in[0,1]$, it follows that, for $\nu$-a.e. $\gamma \in \mathbf{P}_{m_{0}}(M)$,

$$
\begin{aligned}
\left|\psi_{t}(\gamma)\right| & \leq \sum_{i=1}^{k}\left\|\mathcal{T}_{0 \leftarrow s_{i}}^{\sigma^{S_{n}}(-t)(\gamma)}\left(\nabla_{s_{i}} f\right) \underline{\left(\sigma^{S_{n}}(-t)(\gamma)\right)}\right\|_{T_{m_{0}}}\left\|r_{0} S_{n}\left(s_{i}\right)\right\|_{T_{m_{0}}} \\
& \leq \sum_{i=1}^{k}\left\|\left(\nabla_{s_{i}} f\right) \underline{\left(\sigma^{S_{n}}(-t)(\gamma)\right)}\right\|_{T_{\sigma^{S_{n}\left(-t, s_{i}\right)(\gamma)}}}, \quad t \in \mathbb{R} .
\end{aligned}
$$

Since $f \in C^{\infty}\left(M^{k}\right)$, and $M$ is compact, there exists $C_{4}>0$ such that

$$
\left|\psi_{t}(\gamma)\right| \leq C_{4}, \quad \nu \text {-a.e. } \gamma \in \mathbf{P}_{m_{0}}(M), t \in \mathbb{R} .
$$

Furthermore, in virtue of [9], Theorem 4.1 (iii),

$$
\psi_{t} \underset{t \rightarrow 0}{\longrightarrow} \psi_{0} \quad \nu \text {-a.e. }
$$

Step 3. The aim of this step is to verify the existence of $\left.\frac{d}{d t}\right|_{0} \psi_{t}$ in $L^{2}(\nu)$. To this end, fix $i \in\{1, \ldots, k\}$. Since $\left(\nabla_{s_{i}} f\right)^{v}$ is then a smooth function on $M^{k}$, from 9], Section 5, and 4], Lemma 9.1, it follows that

$$
\left.\frac{d}{d t}\right|_{0}\left(\left(\nabla_{s_{i}} f\right) \underline{\left(\sigma^{S_{n}}(-t)(\gamma)\right)}\right)^{v} \text { exists in } L^{2}(\nu), \quad v \in\{1, \ldots, N\} .
$$

Furthermore, there is a $C_{5}>0$ such that

$$
\left|\left(\nabla_{s_{i}} f\right)^{v}\right| \leq C_{5}, \quad v \in\{1, \ldots, N\} .
$$

By [4, Corollary 4.2 and inequalities (i) as well as (ii) of Lemma 4.1 of the same reference, for all $v \in\{1, \ldots, N\}$,

$$
\begin{aligned}
\left.\frac{d}{d t}\right|_{0} & \left(\mathcal{T}_{s_{i} \leftarrow 0}^{\sigma^{S_{n}}(-t)(\gamma)} r_{0} S_{n}\left(s_{i}\right)\right)^{v} \\
= & \left.\frac{d}{d t}\right|_{0}\left(r_{L(\gamma(-t, j, i))}\left(s_{i}\right) S_{n}\left(s_{i}\right)\right)^{v} \text { exists in } L^{2}(\nu)
\end{aligned}
$$

if $n=j \in\{1, \ldots, d\}$. Even though in [4] the geometric flow is generated by a $C^{1}$ function, for $n=d\left(2^{m}+l-1\right)+j$ with $m \in\{0,1, \ldots\}, l \in\left\{1, \ldots, 2^{m}\right\}, j \in\{1, \ldots, d\}$, we may obtain (4.6) from the above reference by decomposing $S_{n}=S_{n}^{1}+S_{n}^{2}+S_{n}^{3}$ where

$$
\begin{aligned}
S_{n}^{1} & =2^{\frac{m}{2}} \chi_{\left[\frac{l-1}{2^{m}}, 1\right]}(s)\left(s-\frac{l-1}{2^{m}}\right), \\
S_{n}^{2} & =-2^{\frac{m}{2}+1} \chi_{\left[\frac{2 l-1}{2^{m+1}}, 1\right]}(s)\left(s-\frac{2 l-1}{2^{m+1}}\right), \\
S_{n}^{3} & =2^{\frac{m}{2}} \chi_{\left[\frac{l}{2^{m}}, 1\right]}(s)\left(s-\frac{l}{2^{m}}\right) .
\end{aligned}
$$

Moreover, by isometric embedding of $M$ into $\mathbb{R}^{N}$ we verify

$$
\begin{aligned}
\left|\left(\mathcal{T}_{s_{i} \leftarrow 0} r_{0} S_{n}\left(s_{i}\right)\right)^{v}\right| & \leq\left|\mathcal{T}_{s_{i} \leftarrow 0} r_{0} S_{n}\left(s_{i}\right)\right|_{\mathbb{R}^{N}} \\
& =\left\|\mathcal{T}_{s_{i} \leftarrow 0} r_{0} S_{n}\left(s_{i}\right)\right\|_{T_{\cdot\left(s_{i}\right)}} \\
& =\left|S_{n}\left(s_{i}\right)\right|_{\mathbb{R}^{d}} \\
& \leq 1, \quad v \in\{1, \ldots, N\} .
\end{aligned}
$$

Introduce

$$
\left.\alpha_{t}^{v}:=\left(\left(\nabla_{s_{i}} f\right) \underline{\left(\sigma^{S_{n}}(-t)(\gamma)\right.}\right)\right)^{v}, \quad \beta_{t}^{v}:=\left(\mathcal{T}_{s_{i} \leftarrow 0}^{\sigma^{S_{n}}(-t)(\gamma)} r_{0} S_{n}\left(s_{i}\right)\right)^{v},
$$


$t \in \mathbb{R}, v \in\{1, \ldots, N\}$. The existence of

$$
\frac{d}{d t}\left|\sum_{0}^{N}\left(\left(\nabla_{s_{i}} f\right)\left(\sigma^{S_{n}}(-t)(\gamma)\right)\right)^{v}\left(\mathcal{T}_{s_{i} \leftarrow 0}^{\sigma^{S_{n}}(-t)(\gamma)} r_{0} S_{n}\left(s_{i}\right)\right)^{v}=\frac{d}{d t}\right| \sum_{0}^{N} \alpha_{v=1}^{v} \beta_{t}^{v}
$$

in $L^{2}(\nu)$ follows from (4.4)-(4.7), $\alpha_{t}^{v} \underset{t \rightarrow 0}{\longrightarrow} \alpha_{0}^{v} \nu$-a.e., and the inequality

$$
\begin{aligned}
\left\|\frac{\sum_{v=1}^{N} \alpha_{t}^{v} \beta_{t}^{v}-\sum_{v=1}^{N} \alpha_{0}^{v} \beta_{0}^{v}}{t}-\sum_{v=1}^{N}\left(\dot{\alpha_{0}^{v}} \beta_{0}^{v}+\alpha_{0}^{v} \dot{\beta_{0}^{v}}\right)\right\|_{L^{2}(\nu)} \\
\leq \sum_{v=1}^{N}\left\|\left(\frac{\alpha_{t}^{v}-\alpha_{0}^{v}}{t}-\dot{\alpha_{0}^{v}}\right) \beta_{0}^{v}\right\|_{L^{2}(\nu)}+\sum_{v=1}^{N}\left\|\left(\frac{\beta_{t}^{v}-\beta_{0}^{v}}{t}-\dot{\beta_{0}^{v}}\right) \alpha_{t}^{v}\right\|_{L^{2}(\nu)} \\
\quad+\sum_{v=1}^{N}\left\|\left(\alpha_{t}^{v}-\alpha_{0}^{v}\right) \dot{\beta_{0}^{v}}\right\|_{L^{2}(\nu)} \\
\leq \sum_{v=1}^{N}\left\|\frac{\alpha_{t}^{v}-\alpha_{0}^{v}}{t}-\dot{\alpha_{0}^{v}}\right\|_{L^{2}(\nu)}\left\|\beta_{0}^{v}\right\|_{L^{\infty}(\nu)}+C_{5} \sum_{v=1}^{N}\left\|\frac{\beta_{t}^{v}-\beta_{0}^{v}}{t}-\dot{\beta_{0}^{v}}\right\|_{L^{2}(\nu)} \\
\quad+\sum_{v=1}^{N}\left\|\left(\left(\alpha_{t}^{v}-\alpha_{0}^{v}\right) \dot{\beta}_{0}^{v}\right)^{2}\right\|_{L^{1}(\nu)}^{1 / 2}, \quad v \in\{1, \ldots, N\} ;
\end{aligned}
$$

note that, by (4.5) $),\left(\left(\alpha_{t}^{v}-\alpha_{0}^{v}\right) \dot{\beta}_{0}^{v}\right)^{2} \in L^{1}(\nu)$ is dominated by $4 C_{5}^{2}\left(\dot{\beta}_{0}^{v}\right)^{2} \in L^{1}(\nu)$, $v \in\{1, \ldots, N\}$. Finally, by isometry

$$
\left.\left.\frac{d}{d t}\right|_{0} \psi_{t}(\gamma)=\left.\sum_{i=1}^{k} \frac{d}{d t}\right|_{0}\left\langle\left(\nabla_{s_{i}} f\right) \underline{\left(\sigma^{S_{n}}(-t)(\gamma)\right.}\right), \mathcal{T}_{s_{i} \leftarrow 0}^{\sigma^{S_{n}}(-t)(\gamma)} r_{0} S_{n}\left(s_{i}\right)\right\rangle_{T_{\sigma S_{n}\left(-t, s_{i}\right)(\gamma)}}
$$

exists $L^{2}(\nu)$.

Step 4. Having in mind that $\varphi_{0} \equiv 1$ and that $\left(\left(\psi_{t}-\psi_{0}\right) \dot{\varphi}_{0}\right)^{2} \in L^{1}(\nu)$ is dominated by $4 C_{4}^{2}\left(\dot{\varphi_{0}}\right)^{2} \in L^{1}(\nu)$, the existence of $\left.\frac{d}{d t}\right|_{0} \varphi_{t}$ and $\left.\frac{d}{d t}\right|_{0} \psi_{t}$ in $L^{2}(\nu)$ (cf. Steps 1 and 3 ), relations (4.2), (4.3), and

$$
\begin{aligned}
& \left\|\frac{\varphi_{t} \psi_{t}-\varphi_{0} \psi_{0}}{t}-\dot{\varphi}_{0} \psi_{0}-\varphi_{0} \dot{\psi_{0}}\right\|_{L^{2}(\nu)} \\
& \quad \leq C_{4}\left\|\frac{\varphi_{t}-\varphi_{0}}{t}-\dot{\varphi}_{0}\right\|_{L^{2}(\nu)}+\left\|\frac{\psi_{t}-\psi_{0}}{t}-\dot{\psi}_{0}\right\|_{L^{2}(\nu)}+\left\|\left(\left(\psi_{t}-\psi_{0}\right) \dot{\varphi}_{0}\right)^{2}\right\|_{L^{1}(\nu)}^{1 / 2}
\end{aligned}
$$

imply the existence of $\left.\frac{d}{d t}\right|_{0}\left\{\partial_{S_{n}} F\left(\sigma^{S_{n}}(-t)\right) \frac{d \nu \circ \sigma^{S_{n}}(-t)}{d \nu}\right\}$ in $L^{2}(\nu)$.

Step 5. As shown, for example in [11], 4.1, it holds that $\partial_{S_{n}} \eta=\left.\frac{d}{d t}\right|_{0} \eta\left(\sigma^{S_{n}}(-t)\right)$, whenever $\eta \in D(\mathbf{D}) \subseteq D\left(\partial_{S_{n}}\right)$ and the $\operatorname{limit}_{\lim _{t \rightarrow 0}}\left(\eta\left(\sigma^{S_{n}}(t)\right)-\eta\right)$ exists in $L^{2}(\nu)$. Since $F \in Y$ belongs to $D\left(\mathbf{D}^{2}\right)$ (see [9], Section 6), we have $\partial_{S_{n}} F=\left(\mathbf{D}_{1} F\right)^{j} \in D(\mathbf{D})$ if $n=j \in\{1, \ldots, d\}$ and we have $\partial_{S_{n}} F=2^{m / 2}\left(-\mathbf{D}_{\frac{l-1}{2^{m}}} F+2 \mathbf{D}_{\frac{2 l-1}{2^{m+1}}} F-\mathbf{D}_{\frac{l}{2^{m}}} F\right)^{j} \in$ $D(\mathbf{D})$ if $n=d\left(2^{m}+l-1\right)+j$ for $m \in\{0,1, \ldots\}, l \in\left\{1, \ldots, 2^{m}\right\}, j \in\{1, \ldots, d\}$. Therefore, relation (4.1) is a consequence of Step 4, in particular, of (4.8).

Let $(A, D(A))$ denote the generator of $(\mathcal{E}, D(\mathcal{E}))$. Fix a version $\bar{H}$ of the map $\gamma \rightarrow \mathcal{T}_{. \leftarrow 0}^{\gamma}$. Using B.K. Driver's geometrical notation (see [4], Definition 6.2 and 
Theorem 9.1), we introduce

$$
z_{n}(\gamma)=\frac{1}{2} \int_{0}^{1}\left(\operatorname{Ric}_{\bar{H} \gamma}\left\langle S_{n}\right\rangle+\hat{\Theta}_{\bar{H} \gamma}\left\langle S_{n}\right\rangle\right) \cdot d x+\int_{0}^{1} S_{n}^{\prime} \cdot d x, \quad n \in \mathbb{N},
$$

where $\gamma=I(x) \in \mathbf{P}_{m_{0}}(M)$ for $\mu$-a.e. $x \in X$.

Theorem 4.2. Let $F \in Y$. We have $F \in D(A)$ and

$$
A F=\sum_{n=1}^{\infty} \lambda_{n}\left\{\partial_{S_{n}} \partial_{S_{n}} F+z_{n} \partial_{S_{n}} F\right\} \quad \nu \text {-a.e. }
$$

Proof. Let $G \in Y$. From (3.2), we obtain

$$
\mathcal{E}(F, G)=\left.\int \sum_{n=1}^{\infty} \lambda_{n} \frac{d}{d t}\right|_{0} G\left(\sigma^{S_{n}}(t)\right) \partial_{S_{n}} F d \nu .
$$

Taking into consideration that $\left.\frac{d}{d t}\right|_{0} G\left(\sigma^{S_{n}}(t)\right)$ exists in $L^{2}(\nu)$, that $\partial_{S_{n}} F=$ $\left\langle S_{n}, \mathbf{D} F\right\rangle_{\mathbb{H}} \in L^{2}(\nu)$, and that the sum in (4.9) is, by Lemma 2.1] actually a finite one, we obtain

$$
\mathcal{E}(F, G)=\left.\frac{d}{d t}\right|_{0} \int \sum_{n=1}^{\infty} \lambda_{n} G\left(\sigma^{S_{n}}(t)\right) \partial_{S_{n}} F d \nu
$$

Under the substitution $\gamma \rightarrow \sigma^{S_{n}}(-t)(\gamma)$, we get

$$
\begin{aligned}
\mathcal{E}(F, G) & =\left.\frac{d}{d t}\right|_{0} \int G \cdot \sum_{n=1}^{\infty} \lambda_{n} \partial_{S_{n}} F\left(\sigma^{S_{n}}(-t)\right) d \nu \circ \sigma^{S_{n}}(-t) \\
& =\left.\frac{d}{d t}\right|_{0} \int G \cdot \sum_{n=1}^{\infty} \lambda_{n} \partial_{S_{n}} F\left(\sigma^{S_{n}}(-t)\right) \frac{d \nu \circ \sigma^{S_{n}}(-t)}{d \nu} d \nu .
\end{aligned}
$$

From Lemma 4.1, it can be concluded that

$$
\mathcal{E}(F, G)=\left.\int G \cdot \sum_{n=1}^{\infty} \lambda_{n} \frac{d}{d t}\right|_{0}\left\{\partial_{S_{n}} F\left(\sigma^{S_{n}}(-t)\right) \frac{d \nu \circ \sigma^{S_{n}}(-t)}{d \nu}\right\} d \nu
$$

again, take into consideration that the above sum is actually a finite one. Let $G^{*}$ be an arbitrary function belonging to $D(\mathcal{E})$. As $Y$ is by Theorem 2.2 dense in $D(\mathcal{E})$ with respect to $\mathcal{E}_{1}^{1 / 2}$-norm, we find a sequence $G_{m} \in Y, m \in \mathbb{N}$, with $G_{m} \underset{m \rightarrow \infty}{\longrightarrow} G^{*}$ in $\mathcal{E}_{1}^{1 / 2}$-norm. In particular, $G_{m} \underset{m \rightarrow \infty}{\longrightarrow} G^{*}$ in $L^{2}(\nu)$. On account of $\left|\mathcal{E}\left(G_{m}-G^{*}, F\right)+\left(G_{m}-G^{*}, F\right)_{L^{2}(\nu)}\right|=\left|\mathcal{E}_{1}\left(G_{m}-G^{*}, F\right)\right| \underset{m \rightarrow \infty}{\longrightarrow} 0$, we verify $\mathcal{E}\left(G_{m}, F\right) \underset{m \rightarrow \infty}{\longrightarrow} \mathcal{E}\left(G^{*}, F\right)$. Now, relation 4.10) and Lemma 4.1 yield

$$
\mathcal{E}\left(F, G^{*}\right)=\left.\int G^{*} \cdot \sum_{n=1}^{\infty} \lambda_{n} \frac{d}{d t}\right|_{0}\left\{\partial_{S_{n}} F\left(\sigma^{S_{n}}(-t)\right) \frac{d \nu \circ \sigma^{S_{n}}(-t)}{d \nu}\right\} d \nu .
$$

Therefore, we have $F \in D(A)$ and

$$
A F=-\left.\sum_{n=1}^{\infty} \lambda_{n} \frac{d}{d t}\right|_{0}\left\{\partial_{S_{n}} F\left(\sigma^{S_{n}}(-t)\right) \frac{d \nu \circ \sigma^{S_{n}}(-t)}{d \nu}\right\} \quad \nu \text {-a.e. }
$$


From Lemma 4.1, it can be deduced that

$$
A F=\sum_{n=1}^{\infty} \lambda_{n}\left\{\partial_{S_{n}} \partial_{S_{n}} F-\left.\frac{d}{d t}\right|_{0} \frac{d \nu \circ \sigma^{S_{n}}(-t)}{d \nu} \cdot \partial_{S_{n}} F\right\} \quad \nu \text {-a.e. }
$$

According to 4, Lemma 9.2, or 9], proof of Theorem 5.1, it holds that

$$
\left.\frac{d}{d t}\right|_{0} \frac{d \nu \circ \sigma^{S_{n}}(-t)}{d \nu}=-z_{n} \quad \nu \text {-a.e. }
$$

From (4.11), it follows that

$$
A F=\sum_{n=1}^{\infty} \lambda_{n}\left\{\partial_{S_{n}} \partial_{S_{n}} F+z_{n} \partial_{S_{n}} F\right\} \quad \nu \text {-a.e. }
$$

Remark. (5) Keeping in mind that, for $F \in Y$, the sum $\sum_{n=1}^{\infty} \lambda_{n} z_{n} \partial_{S_{n}} F$ is actually a finite sum (cf. Lemma 2.1), we can verify the following identity:

$$
\begin{aligned}
\sum_{n=1}^{\infty} \lambda_{n} z_{n} \partial_{S_{n}} F(\gamma) \\
=\sum_{n=1}^{\infty} \lambda_{n}\left\{\frac{1}{2} \int_{0}^{1}\left(\operatorname{Ric}_{\bar{H} \gamma}\left\langle S_{n}\right\rangle+\hat{\Theta}_{\bar{H} \gamma}\left\langle S_{n}\right\rangle\right) \cdot d x+\int_{0}^{1} S_{n}^{\prime} \cdot d x\right\} \partial_{S_{n}} F(\gamma) \\
=\frac{1}{2} \int_{0}^{1}\left(\operatorname{Ric}_{\bar{H} \gamma}\left\langle\sum \lambda_{n} \partial_{S_{n}} F S_{n}\right\rangle+\hat{\Theta}_{\bar{H} \gamma}\left\langle\sum \lambda_{n} \partial_{S_{n}} F S_{n}\right\rangle\right) \cdot d x \\
\quad+\int_{0}^{1}\left(\sum \lambda_{n} \partial_{S_{n}} F S_{n}^{\prime}\right) \cdot d x \\
=\frac{1}{2} \int_{0}^{1}\left(R i c_{\bar{H} \gamma}\langle(\mathbf{A D} F)(s)(\gamma)\rangle+\hat{\Theta}_{\bar{H} \gamma}\langle(\mathbf{A D} F)(s)(\gamma)\rangle\right) \cdot d x_{s} \\
\quad+\int_{0}^{1}(\mathbf{A D} F)^{\prime}(s)(\gamma) \cdot d x_{s}
\end{aligned}
$$

$\nu$-a.e., where $\gamma=I(x) \in \mathbf{P}_{m_{0}}(M)$ for $\mu$-a.e. $x \in X$.

\section{LOCAL SECOND MOMENT}

We begin this section with a general lemma. For a moment, we introduce a new setting more general than the situation in Sections 1-4. Let $E$ be a Hausdorff topological space and let $\mathcal{B}(E)$ denote its Borel $\sigma$-algebra. Suppose, furthermore, that $\mathcal{B}(E)=\sigma(C(E))$ where $C(E)$ denotes the set of all continuous functions on $E$. Let $\nu$ be a probability measure on $(E, \mathcal{B})$ and $(\mathcal{E}, D(\mathcal{E}))$ a quasi-regular Dirichlet form on $L^{2}(E, \nu)$. Let $(A, D(A))$ denote the generator of $(\mathcal{E}, D(\mathcal{E}))$ and assume that there exists a subspace $\mathbb{G} \subseteq D(A)$, dense in $\left(D(\mathcal{E}), \mathcal{E}_{1}^{1 / 2}\right)$, such that $g \in \mathbb{G}$ implies that $g^{2} \in D(A)$. Then, according to [3], Proposition I.4.1.3 and Corollary I.4.2.3, there exists a unique carré du champ operator $\Gamma: D(\mathcal{E}) \times D(\mathcal{E}) \rightarrow L^{1}(E, \nu)$. In particular, for all $f, g \in D(\mathcal{E}) \cap L^{\infty}(E, \nu)$, it holds that

$$
\int g \Gamma(f, f) d \nu=-\mathcal{E}\left(g, f^{2}\right)+2 \mathcal{E}(f g, f) .
$$


Let $\mathbf{M}=\left(\Omega, \mathcal{F},\left(X_{t}\right)_{t \geq 0},\left(P_{x}\right)_{x \in E}\right)$ denote the associated right process, set $\mathbf{1}(x)=1$, $x \in E$, and define

$$
T_{t} f(x):=\int f(y) P_{x}\left(X_{t} \in d y\right), \quad t \geq 0, x \in E, f \in L^{2}(E, \nu) .
$$

Proposition 5.1. Suppose $\mathbf{1} \in \mathbb{G}, T_{t} \mathbf{1}=\mathbf{1}$ for all $t \geq 0$, and $f g \in D(\mathcal{E}) \cap L^{\infty}(E, \nu)$ if $f \in D(\mathcal{E}) \cap L^{\infty}(E, \nu)$ and $g \in \mathbb{G}$. Then we have

$$
\begin{aligned}
& \lim _{t \rightarrow 0} \frac{1}{t} \int(f(y)-f(\cdot))^{2} P .\left(X_{t} \in d y\right) \\
& \quad=\Gamma(f, f) \quad \text { weakly in } L^{1}(E, \nu), f \in D(\mathcal{E}) \cap L^{\infty}(E, \nu) .
\end{aligned}
$$

Proof. Step 1. Let $I d$ denote the identity in $L^{2}(E, \nu)$ and let $f \in D(\mathcal{E}) \cap L^{\infty}(E, \nu)$ and $g \in \mathbb{G}$. Since $g \in D(A) \subseteq L^{2}(E, \nu), f^{2} \in L^{\infty}(E, \nu) \subseteq L^{2}(E, \nu)$, and $T_{t}-I d$ is a selfadjoint operator in $L^{2}(E, \nu), t>0$, we can conclude

$$
\begin{aligned}
-\mathcal{E}\left(g, f^{2}\right) & =\left(A g, f^{2}\right)_{L^{2}(E, \nu)} \\
& =\left(\lim _{t \rightarrow 0} \frac{\left(T_{t}-I d\right) g}{t}, f^{2}\right)_{L^{2}(E, \nu)} \\
& =\lim _{t \rightarrow 0}\left(\frac{\left(T_{t}-I d\right) g}{t}, f^{2}\right)_{L^{2}(E, \nu)} \\
& =\lim _{t \rightarrow 0}\left(g, \frac{\left(T_{t}-I d\right) f^{2}}{t}\right)_{L^{2}(E, \nu)} .
\end{aligned}
$$

Let $\left(E_{\lambda}\right)_{\lambda \geq 0}$ denote the (right continuous) resolution of the identity with respect to $-A$, i.e.,

$$
-A f=\int_{[0, \infty)} \lambda d E_{\lambda} f, \quad f \in D(A),
$$

recall that $A$ is a nonpositive definite selfadjoint operator in $L^{2}(E, \nu)$. The closed form $(\mathcal{E}, D(\mathcal{E}))$ has, therefore, a representation

$$
\mathcal{E}(f, f)=\int_{[0, \infty)} \lambda d\left\|E_{\lambda} f\right\|_{L^{2}(E, \nu)}^{2}, \quad f \in D(\mathcal{E})
$$

Taking into consideration $-\lambda \leq \frac{e^{-\lambda t}-1}{t} \leq 0$ if $\lambda \geq 0$ and $t>0$, we obtain

$$
\begin{aligned}
\mathcal{E}(f g, f) & =\int_{[0, \infty)} \lambda d\left(E_{\lambda}(f g), E_{\lambda} f\right)_{L^{2}(E, \nu)} \\
& =-\lim _{t \rightarrow 0} \int_{[0, \infty)} \frac{e^{-\lambda t}-1}{t} d\left(E_{\lambda}(f g), E_{\lambda} f\right)_{L^{2}(E, \nu)} \\
& =-\lim _{t \rightarrow 0}\left(\int_{[0, \infty)} d E_{\lambda}(f g), \int_{[0, \infty)} \frac{e^{-\lambda t}-1}{t} d E_{\lambda} f\right)_{L^{2}(E, \nu)} \\
& =-\lim _{t \rightarrow 0}\left(f g, \frac{T_{t} f-f}{t}\right)_{L^{2}(E, \nu)} .
\end{aligned}
$$


Combining $T_{t} \mathbf{1}=\mathbf{1}, t \geq 0$, (5.1), (5.2), and (5.3), we verify

$$
\begin{aligned}
\int g \Gamma(f, f) d \nu & =-\mathcal{E}\left(g, f^{2}\right)+2 \mathcal{E}(f g, f) \\
& =\lim _{t \rightarrow 0} \int g \cdot\left(\frac{T_{t} f^{2}-f^{2}}{t}-2 f \frac{T_{t} f-f}{t}\right) d \nu \\
& =\lim _{t \rightarrow 0} \int g(x) \cdot \frac{1}{t} \int(f(y)-f(x))^{2} P_{x}\left(X_{t} \in d y\right) \nu(d x) .
\end{aligned}
$$

Step 2. Again, let $f \in D(\mathcal{E}) \cap L^{\infty}(E, \nu)$. For $g=\mathbf{1}$, relation (5.4) reduces to

$$
2 \mathcal{E}(f, f)=\lim _{t \rightarrow 0}\left\|\frac{1}{t} \int(f(y)-f(\cdot))^{2} P .\left(X_{t} \in d y\right)\right\|_{L^{1}(E, \nu)} .
$$

Setting

$$
\varphi_{t}:= \begin{cases}\Gamma(f, f) & \text { if } t=0, \\ \frac{1}{t} \int(f(y)-f(\cdot))^{2} P .\left(X_{t} \in d y\right) & \text { if } t>0\end{cases}
$$

from $\varphi_{t}=\frac{T_{t} f^{2}-f^{2}}{t}-2 f \frac{T_{t} f-f}{t}, t>0$, and (5.5) it follows that $\left\|\varphi_{t}\right\|_{L^{1}(E, \nu)}$ is continuous on $[0, \infty)$ and $\lim _{t \rightarrow \infty}\left\|\varphi_{t}\right\|_{L^{1}(E, \nu)}=0$. Thus, the family $\left(\varphi_{t}\right)_{t \geq 0}$ is uniformly bounded in $L^{1}(E, \nu)$. Now the statement of the lemma is a consequence of relation (5.4).

In the remainder of this section, we follow the setting of Sections 1-4. In particular, let $\mathbf{M}=\left(\Omega, \mathcal{F},\left(X_{t}\right)_{t \geq 0},\left(P_{\gamma}\right)_{\gamma \in \mathbf{P}_{m_{0}}(M)}\right)$ denote the right process associated with $(\mathcal{E}, D(\mathcal{E}))$; cf. Theorem 3.5 Furthermore, recall that $x^{v}(p), v \in\{1, \ldots, N\}$, denote the standard coordinates of $p \in M$ embedded in $\mathbb{R}^{N}$. For fixed $s \in[0,1]$ and $v \in\{1, \ldots, N\}$, introduce the function $x_{s}^{v}$ by $x_{s}^{v}(\gamma):=x^{v}(\gamma(s)), \gamma \in \mathbf{P}_{m_{0}}(M)$.

Lemma 5.2. Suppose the validity of relation (3.3), i.e.,

$$
\lambda_{i} \leq c i^{1-\varepsilon}, \quad i \in \mathbb{N}, \quad \text { for some } c>0 \text { and } \varepsilon \in(0,1) .
$$

Then $x_{s}^{v} \in D(\mathcal{E}) \cap L^{\infty}(\nu)$ and there exists $C_{6}>0$ such that

$$
\mathcal{E}\left(x_{s}^{v}, x_{s}^{v}\right) \leq C_{6}
$$

for all $s \in[0,1]$ and $v \in\{1, \ldots, N\}$.

Proof. Let $v \in\{1, \ldots, N\}, s \in[0,1]$, and $s_{n} \in\left\{\frac{l}{2^{m}}: l \in\left\{1, \ldots, 2^{m}\right\}, m \in \mathbb{N}\right\}$, $n \in \mathbb{N}$, be a sequence with $s_{n} \underset{n \rightarrow \infty}{\longrightarrow} s$. Then

$$
x_{s_{n}}^{v} \underset{n \rightarrow \infty}{\longrightarrow} x_{s}^{v} \quad \nu \text {-a.e. }
$$

Furthermore, as $M$ is compact, there is a constant $C_{7}>0$ such that

$$
\left\|x_{s_{n}}^{v}\right\|_{L^{2}(\nu)}^{2} \leq C_{7}
$$

independent of $n \in \mathbb{N}$. As in (3.5)-(3.8), it follows from (3.3) that

$$
\mathcal{E}\left(x_{s_{n}}^{v}, x_{s_{n}}^{v}\right) \leq C_{1},
$$

independent of $n \in \mathbb{N}$, where $C_{1}$ is the constant introduced in (3.7). Now, the above mentioned Banach-Saks property of the Hilbert space $\left(D(\mathcal{E}), \mathcal{E}_{1}^{1 / 2}\right)$ and (5.7)-(15.9) imply $x_{s}^{v} \in D(\mathcal{E}) \cap L^{\infty}(\nu)$ and from the closedness of $(\mathcal{E}, D(\mathcal{E}))$ on $L^{2}(\nu)$, relation (5.6) with $C_{6}:=C_{1}+C_{7}$ can be derived. 
Set $\mathbb{G}:=Y$. According to Theorems 2.2 and $4.2 \mathbb{G}$ is dense in $\left(D(\mathcal{E}), \mathcal{E}_{1}^{1 / 2}\right)$ and we have $\mathbb{G} \subseteq D(A)$. Obviously, $g \in \mathbb{G}$ implies $g^{2} \in \mathbb{G}$. Thus, there exists a carré du champ operator $\Gamma$ and we have (5.1). In order to formulate the following theorem, we notice that, for $h \in L^{\infty}(\nu)$,

$$
\begin{aligned}
\int|h| \cdot \Gamma\left(x_{s}^{v}, x_{s}^{v}\right) d \nu & \leq 2\|h\|_{L^{\infty}(\nu)} \cdot \mathcal{E}\left(x_{s}^{v}, x_{s}^{v}\right) \\
& \leq 2 C_{6}\|h\|_{L^{\infty}(\nu)},
\end{aligned}
$$

independent of $s \in[0,1]$ and $v \in\{1, \ldots, N\}$ (cf. (5.6)), which implies

$$
\int_{\gamma \in \mathbf{P}_{m_{0}}}|h| \cdot \sum_{v=1}^{N} \int_{s \in[0,1]} \Gamma\left(x_{s}^{v}(\gamma), x_{s}^{v}(\gamma)\right) d s \nu(d \gamma) \leq 2 N C_{6}\|h\|_{L^{\infty}(\nu)} .
$$

Theorem 5.3. Suppose that relation (3.3) is valid, i.e.,

$$
\lambda_{i} \leq c i^{1-\varepsilon}, \quad i \in \mathbb{N}, \quad \text { for some } c>0 \text { and } \varepsilon \in(0,1) .
$$

For all $h \in L^{\infty}(\nu)$, we have

$$
\begin{aligned}
& \lim _{t \rightarrow 0} \int h(\tau) \cdot \frac{1}{t} \int \sum_{v=1}^{N}\left\|x^{v}(\gamma)-x^{v}(\tau)\right\|_{L^{2}([0,1], d s)}^{2} P_{\tau}\left(X_{t} \in d \gamma\right) \nu(d \tau) \\
& =\int h(\tau) \cdot \sum_{v=1}^{N} \int_{s \in[0,1]} \Gamma\left(x_{s}^{v}, x_{s}^{v}\right) d s \nu(d \tau) .
\end{aligned}
$$

Proof. In order to apply Proposition [5.1, we note that $\mathbf{1} \in \mathbb{G}, P_{\tau}\left(X_{t} \in \mathbf{P}_{m_{0}}(M)\right)=$ $1, t \geq 0, \tau \in \mathbf{P}_{m_{0}}(M)$, and that because of $\mathbb{G}=Y \subseteq D(\mathcal{E}) \cap L^{\infty}(\nu)$, we have $f g \in D(\mathcal{E}) \cap L^{\infty}(\nu)$ if $f \in D(\mathcal{E}) \cap L^{\infty}(\nu)$ and $g \in \mathbb{G}$.

By virtue of Lemma 5.2 and Proposition 5.1 for all $s \in[0,1], v \in\{1, \ldots, N\}$, and $h \in L^{\infty}(\nu)$, it holds that

$$
\lim _{t \rightarrow 0} \int h \cdot \frac{1}{t} \int\left(x_{s}^{v}(\gamma)-x_{s}^{v}\right)^{2} P .\left(X_{t} \in d \gamma\right) d \nu=\int h \Gamma\left(x_{s}^{v}, x_{s}^{v}\right) d \nu .
$$

Since $\left\|\frac{1}{t} \int\left(x_{s}^{v}(\gamma)-x_{s}^{v}\right)^{2} P .\left(X_{t} \in d \gamma\right)\right\|_{L^{1}(\nu)}$ is bounded for $t>0$ (cf. proof of Proposition [5.1), it can be concluded from dominated convergence that, for all $v \in\{1, \ldots, N\}$,

$$
\begin{aligned}
& \lim _{t \rightarrow 0} \int_{s \in[0,1]} \int h \cdot \frac{1}{t} \int\left(x_{s}^{v}(\gamma)-x_{s}^{v}\right)^{2} P .\left(X_{t} \in d \gamma\right) d \nu d s \\
& =\int_{s \in[0,1]} \int h \Gamma\left(x_{s}^{v}, x_{s}^{v}\right) d \nu d s, \quad h \in L^{\infty}(\nu) .
\end{aligned}
$$

Relation (5.11) is now a direct consequence of (5.10) and Fubini's theorem.

Remark. (6) Condition (3.3) in Proposition 3.3, Lemma 5.2, and Theorem5.3 can be weakened. Recalling (3.6) it turns out that it is sufficient to require

$$
\sum_{m=1}^{\infty} \lambda_{d 2^{m}} 2^{-m}<\infty
$$

instead of (3.3). 


\section{ACKNOWLEDGEMENTS}

The author is grateful to the colleagues of the Department of Mathematics at Northwestern University for their hospitality. The author would especially like to thank Elton P. Hsu.

\section{REFERENCES}

[1] S. AIDA, Logarithmic derivatives of heat kernels and logarithmic Sobolev inequalities with unbounded diffusion coefficients on loop spaces. J. Funct. Anal., 174 (2000), 430-477. MR 2001i:60024

[2] S. Albeverio, M. Röckner, Stochastic differential equations in infinite dimensions: solutions via Dirichlet forms, Probab. Th. Rel. Fields, 89 (1991), 347-386. MR 92k:60123

[3] N. Bouleau, F. Hirsch, Dirichlet forms and analysis on Wiener space. Berlin, New York: Walter de Gruyter, 1991. MR 93e:60107

[4] B.K. Driver, A Cameron-Martin type quasi-invariance theorem for Brownian motion on a compact Riemannian manifold. J. Funct. Anal., 110 (1992), 272-376. MR 94a:58214

[5] B.K. Driver, M. Röckner, Construction of diffusions on path and loop spaces of compact Riemannian manifolds. C.R. Acad. Sci. Paris, Série I, 315 (1992), 603-608. MR 93f:58246

[6] A. Eberle, Diffusions on path and loop spaces: existence, finite dimensional approximation and Hölder continuity, Probab. Th. Rel. Fields, 109 (1997), 77-99. MR 99k:58194

[7] J. Eells, D. Elworthy, Stochastic dynamical systems. In: Control theory and topics in functional analysis, III, Vienna: International Atomic Energy Agency, 1976. MR 58:24390

[8] M. Fukushima, Y. Oshima, M. Takeda, Dirichlet forms and symmetric Markov processes. Berlin, New York: Walter de Gruyter, 1994. MR 96f:60126

[9] E. P. Hsu, Quasi-invariance of the Wiener measure on the path space over a compact Riemannian manifold. J. Funct. Anal., 134 (1995), 417-450. MR 97c:58163

[10] S. Kusuoka, Dirichlet forms and diffusion processes on Banach spaces. J. Fac. Sci. Univ. Tokyo Sect. IA Math. 29 (1982), 79-95. MR 83h:60082

[11] J.-U. Löвus, A Flat Copy of the Ornstein-Uhlenbeck Operator on the Path Space over a Riemannian Manifold, Infinite Dimensional Analysis, Quantum Probability and Related Topics 5, No. 3 (2002), 351-394. MR 2003g:58059

[12] P. Malliavin, Formulae de la moyenne, calcul de perturbations et theórème d'annulation pour les formes harmoniques. J. Funct. Anal., 17 (1974), 274-291. MR 52:6791

[13] Z.M. MA, M. RöcKNeR, An introduction to the theory of (non-symmetric) Dirichlet forms. Berlin: Springer, 1992. MR 94d:60119

[14] N. Okada, On the Banach-Saks property. Proc. Japan Acad. Ser. A Math. Sci., 60 (1984), 246-248. MR 86g:46027

[15] M. Röckner, B. Schmuland, Tightness of general $C_{1, p}$ capacities on Banach space. J. Funct. Anal., 108 (1992), 1-12. MR 93g:28011

[16] M. RöCKNER, T.S. Zhang, Finite dimensional approximation of diffusion processes on infinite dimensional spaces. Stochastics Stochastics Rep., 57 (1996), 37-55. MR 98e:60128

Department of Mathematics and Computer Science, University of Jena, D-07740 Jena, Germany

Current address: Department of Mathematical Sciences, University of Delaware, 501 Ewing

Hall, Newark, Delaware 19716-2553

E-mail address: loebus@math.udel.edu 